\title{
PAYLOAD MAXIMIZATION FOR MOBILE FLEXIBLE MANIPULATORS IN ENVIRONMENT WITH AN OBSTACLE
}

\author{
HAMIDREZA HEIDARI \\ College of Mechanical Engineering, Malayer University, Malayer, Iran \\ e-mail:hr.heidari@malayeru.ac.ir \\ Mohammad Haghpanahi, Moharam Habibnejad Korayem \\ College of Mechanical Engineering, Iran University of Science and Technology, Tehran, Iran
}

\begin{abstract}
A mobile flexible manipulator is developed in order to achieve high performance requirements such as high-speed operation, increased high payload to mass ratio, less weight, and safer operation due to reduced inertia. Hence, this paper presents a method for finding the Maximum Allowable Dynamic Load (MADL) of geometrically nonlinear flexible link mobile manipulators. The full dynamic model of a wheeled mobile base and the mounted flexible manipulator is considered with respect to dynamics of non-holonomic constraint in environment including an obstacle. In dynamical analysis, an efficient model is employed to describe the treatment of a flexible structure in which both the geometric elastic nonlinearity and the foreshortening effects are considered. Then, a path planning algorithm is developed to find the maximum payload that the optimal strategy is based on the indirect solution to the open-loop optimal control problem. In order to verify the effectiveness of the presented algorithm, several simulation studies are carried out for finding the optimal path between two points in the presence of obstacles. The results clearly show the effect of flexibility and the proposed approach on mobile flexible manipulators.
\end{abstract}

Keywords: flexible link, nonholonomic mobile manipulator, optimal control, obstacle, path planning

\section{Introduction}

Mobile manipulators that are required to have a long reach, fast motion and reduced weight typically also possess significant structural flexibility. For example, mobile flexible manipulators have important application in space stations, manufacturing automation, nuclear contaminated environments, and many other areas. A common task for mobile robots is handling heavy loads from one place to another, particularly for wheeled mobile flexible manipulators when operating in high speeds with long arms. For such systems, to make an effective use of robotic systems, it is important to consider the path planning of the system for finding full-load motion in point-to-point maneuvers since it increases the productivity and economic usage of robotic systems. However, kinematic and dynamic analysis of such a nonholonomic wheeled mobile robot (WMR) is challenging due to complex wheel/manipulator interactions, flexibility and kinematic constraints. An efficient model should be employed to describe the treatment of a flexible structure in which both the geometric elastic nonlinearity and the foreshortening effects are considered.

In this investigation, the optimal strategy is based on the indirect solution to the open-loop optimal control problem. In the open loop optimal control, in spite of the closed-loop ones, many difficulties like process nonlinearities and all types of constraints can be explicitly considered because of the off-line computation of optimal trajectories. On the other hand, the indirect solution method appears to be a well suited approach for this kind of problems, which is based 
on Pontryagin's minimum principle. Combining this approach with an iterative algorithm, the optimal paths and maximum load for WMM in the presence of obstacles can be achieved.

Many researchers have studied the problems of mobile manipulators for the last few years. The dynamic model for links in most of these researches is often based on rigid or small deflection theory, but for applications like light-weight links, high-precision elements or high speed maneuver, it is necessary to capture the deflection caused by nonlinear terms. Seraji (1998) reported a simple on-line approach for motion control of mobile manipulators using the augmented Jacobian matrix. Yamamoto and Yun (1994) focused their research on the modeling and compensation of the dynamic interaction between the manipulator and the mobile platform of a mobile manipulator, and developed a coordination algorithm based on the concept of a preferred operating region. Korayem et al. (2012) and Xi and Fenton (1991) designed an algorithm for motion planning of flexible manipulators in quasi-static operations. A concise motion expression for flexible manipulators was developed to reflect the contributions of joint motions and link deflections to the motion of the end effector by three respective Jacobians. It was found that the algorithm was efficient and accurate for motion planning of flexible link manipulators. Damaren and Sharf (1995) presented and classified different types of inertial and geometric nonlinearities in the dynamical equation for flexible multibody systems. They observed that for sufficiently fast maneuvers of the flexible-link manipulators, the ruthlessly linearized approximation completely inadequate.

Several papers tried to give an answer to the path planning problem and calculate the MADL for rigid and flexible manipulators. For instance, Wang et al. (2001) developed an algorithm that maximized the robot payload while taking into account realistic constraints such as joint torque limits and velocity bounds. The governing optimal control problem was converted into a direct, SQP parameter optimization in which the joint trajectories were defined by B-spline polynomials along with a time-scale factor. Park (2003) presented a method for generating the path of a redundant flexible manipulator which significantly reduced residual vibration in the presence of obstacles. The proposed method was based on an optimized path that was constructed from a combined Fourier series and polynomial with coefficients of each harmonic term selected to minimize the residual vibration.

One of the most popular methods for obstacle avoidance is the artificial potential field method (Castro et al. 2002). In contrast to many methods, the robot motion planning through artificial potential fields (APF) is able to take into account simultaneously the problems of obstacle avoidance and trajectory planning. The first use of the APF concept for obstacle avoidance was presented by Khatib (1986). He proposed the force involving an artificial repulsion from the surface which should be non-negative, continuous and differentiable. More recently, a new version such as the repulsive artificial potential field has been proposed (Agirrebeitia et al., 2005). In most of the previous works, flexibility and nonholonomic constraints of the wheeled mobile manipulator in the path planning problem have not been considered. Hence, this paper proposes a method for planning the trajectory of the nonholonomic mobile flexible manipulator for determining the maximum allowable load and considering the effect of flexibility to achieve the specified point to point maneuver in the presence of an obstacle.

\section{Kinematic and dynamic model of the mobile flexible manipulator}

In this Section, for the sake of modeling and analysis, a mobile flexible manipulator comprising a manipulator flexible arm mounted on a nonholonomic mobile base is considered, as shown in Fig. 1. The motion of the system has to be decomposed into the motion of the flexible manipulator and the motion of the base. The unidirectional platform shown in Fig. 2 is a typical example of a nonholonomic WMR which has two rear driving wheels and two castor wheels. 
The two driving wheels are powered by DC motors and have the same wheel radius $r$. The point $P_{b}$ is the origin of WMR axis, which is located at the intersection of the longitudinal $x$-axis and the lateral $y$-axis. $L_{0}$ and $b$ are length and width or WMR body, respectively. The origin of the inertial frame $\{X, Y\}$ is shown as $O$ and as such allows the position of the WMR to be completely specified through the following vector of generalized coordinates with respect to $\{X, Y\}, \mathbf{q}_{b}=\left[X_{b}, Y_{b}, \varphi, \theta_{r}, \theta_{l}\right]$, where $X_{b}$ and $Y_{b}$ are the coordinates of the center of mass. The orientation of the WMR frame from the inertial frame is denoted by $\varphi \cdot \theta_{r}$ and $\theta_{l}$ are the angular displacements of the right and left driving wheel, respectively. Due to the nonholonomic nature of the system, the constraint equation obeys the ideal no-slip condition. The rolling and the knife edge constraint equations for this system can be found in Yamamoto and Yun (1994). In the next Section, these constraints will be explained.

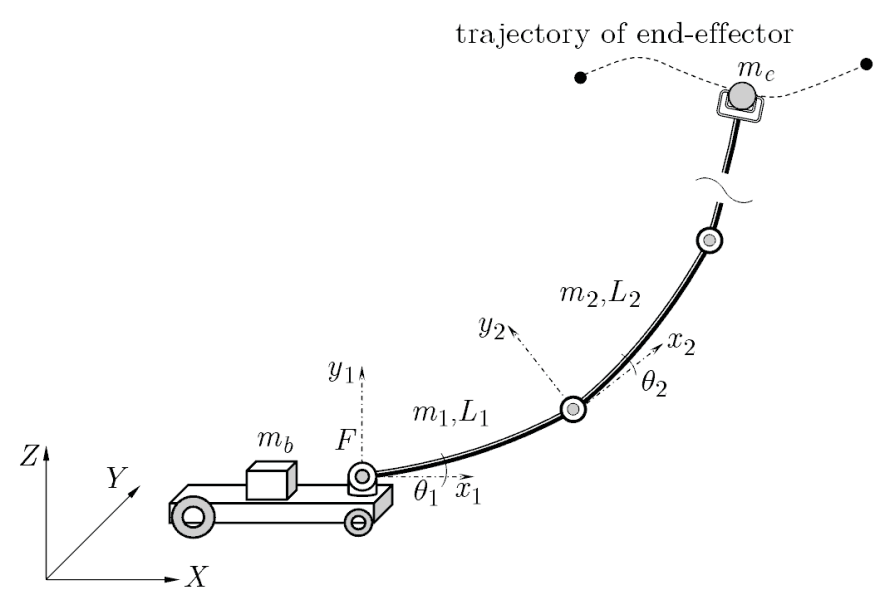

Fig. 1. Schematic view of a mobile flexible manipulator

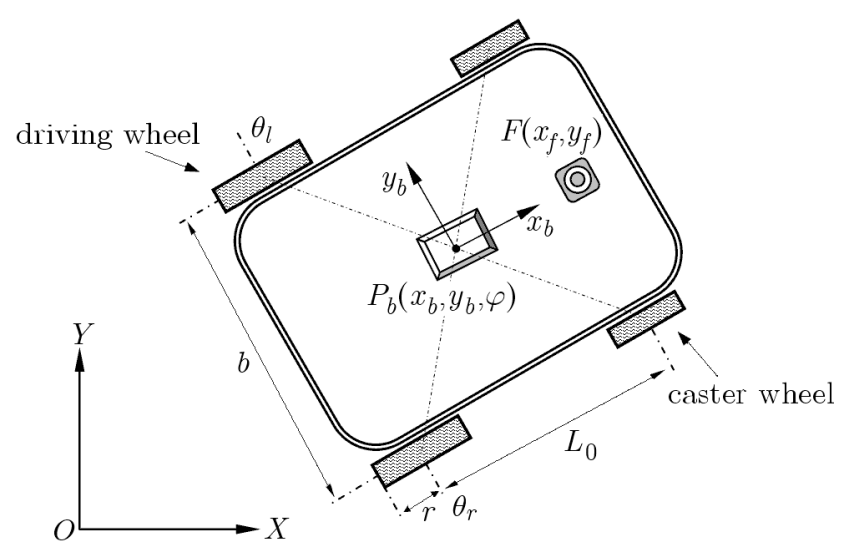

Fig. 2. Nonholonomic wheeled mobile robot platform

The global position vector of the end-effector $\mathbf{r}$ can be defined by appropriately considering the position vector of the corresponding local coordinate in the global reference system as

$$
\mathbf{r}_{i}=\mathbf{r}_{b}+\mathbf{r}_{m / b}=\mathbf{r}_{b}+{ }_{i-1}^{0} \mathbf{r}+\mathbf{R}\left\{\begin{array}{c}
x+u \\
v \\
w
\end{array}\right\}
$$

where $\mathbf{r}_{b}$ is the position of the mobile platform, $\mathbf{R}$ is the transformation matrix and $u, v, w$ denote the longitudinal and transverse displacements.

The analysis of the flexible link can be modeled by slender elastic beams. In this investigation, a more efficient computationally model is employed, in which both the geometric elastic 
nonlinearity and the foreshortening effects are considered. The model takes into account the distinction between the longitudinal displacement due to axial deformation, denoted as $s$, and the longitudinal displacement that can occur due to the foreshortening effect, denoted by $u_{f s}$. The longitudinal displacement caused by transverse deflection of the neutral axis of the beam can be expressed as (Korayem et al., 2012)

$$
u_{f s}=-\frac{1}{2} \int_{0}^{x}\left[\left(\frac{\partial v}{\partial x}\right)^{2}+\left(\frac{\partial w}{\partial x}\right)^{2}\right] d x
$$

The assumed field of displacements for $\mathbf{u}$ can be written as

$$
\mathbf{u}=\left[\begin{array}{c}
u \\
v \\
w
\end{array}\right]=\left[\begin{array}{c}
s+u_{f s} \\
v \\
w
\end{array}\right]
$$

The general expression of the strain energy is written in terms of $s, v$ and $w$, as below (Korayem et al., 2012)

$$
U=\frac{E A}{2} \int_{0}^{l}\left(\frac{\partial s}{\partial x}\right)^{2} d x+\frac{E I}{2} \int_{0}^{l}\left(\frac{\partial^{2} v}{\partial x^{2}}\right)^{2} d x+\frac{E I}{2} \int_{0}^{l}\left(\frac{\partial^{2} w}{\partial x^{2}}\right)^{2} d x
$$

where $E, A, I$, and $l$ denote Young's modulus, cross-sectional area, moment of inertia of the cross section, and length, respectively. This formulation brings nonlinear inertia terms and a constant stiffness matrix in the equations of motion.

The Lagrangian method is utilized to formulate the dynamic equations governing the motion of mobile flexible manipulator systems. In order to derive dynamic equations, the kinetic energy and the potential energy are computed for the entire system. The kinetic energy for the overall system is obtained by computing the kinetic energy for each element $i j$ and then by summing over all the elements. The potential energy of the manipulator is obtained by computing the strain energy for each element $i j$ due to elasticity and gravity of any link. After calculation of these energies, by applying the Lagrangian multipliers procedure and performing some algebraic manipulations, the compact form of the governing equations of a two-link flexible mobile manipulator can be obtained from

$$
\left[\begin{array}{ccc}
M_{b b} & M_{b m} & M_{b f} \\
M_{m b} & M_{m m} & M_{m f} \\
M_{f b} & M_{f m} & M_{f f}
\end{array}\right]\left[\begin{array}{c}
\ddot{q}_{b} \\
\ddot{q}_{m} \\
\ddot{q}_{f}
\end{array}\right]+\left[\begin{array}{c}
C_{b}\left(q_{b}, q_{m}, q_{f}, \dot{q}_{b}, \dot{q}_{m}, \dot{q}_{f}\right) \\
C_{m}\left(q_{b}, q_{m}, q_{f}, \dot{q}_{b}, \dot{q}_{m}, \dot{q}_{f}\right) \\
C_{f}\left(q_{b}, q_{m}, q_{f}, \dot{q}_{b}, \dot{q}_{m}, \dot{q}_{f}\right)
\end{array}\right]+\left[\begin{array}{c}
G_{b}\left(q_{b}, q_{m}, q_{f}\right) \\
G_{m}\left(q_{b}, q_{m}, q_{f}\right) \\
G_{f}\left(q_{b}, q_{m}, q_{f}\right)
\end{array}\right]=\left[\begin{array}{c}
F_{b}-A^{\mathrm{T}}(q) \lambda \\
F_{m} \\
0
\end{array}\right]
$$

where $\mathbf{M}$ is the nonlinear mass matrix, $\mathbf{C}$ is the vector of Coriolis and centrifugal forces, $\mathbf{G}$ describes the gravity effects, and $\mathbf{A}$ denotes the nonholonomic constraints. The generalized coordinates $\mathbf{q}$ and the generalized force $\mathbf{F}$ are the following vectors

$$
\begin{aligned}
& \mathbf{q}=\left[q_{b}, q_{m}, q_{f}\right]=\left[X_{f}, Y_{f}, \varphi, \theta_{r}, \theta_{l}, \theta_{1}, \theta_{2}, q_{f 1}, \ldots, q_{f n}\right] \\
& \mathbf{F}=\left[F_{b}, F_{m}, 0\right]=\left[0,0,0, \tau_{w r}, \tau_{w l}, \tau_{1}, \tau_{2}, 0, \ldots, 0\right]
\end{aligned}
$$

The WMRs are called nonholonomic mobile robots because of their no-slip kinematic constraints. The vehicle is prevented from sliding sideways relative to its instantaneous heading, and each drive wheel is assumed to roll without slipping. These three independent nonholonomic constraints are represented as

$$
\begin{aligned}
& \dot{Y}_{f} \cos \varphi-\dot{X}_{f} \sin \varphi-d \dot{\varphi}=0 \\
& \dot{Y}_{f} \sin \varphi+\dot{X}_{f} \cos \varphi+b \dot{\varphi}=r \dot{\theta}_{r} \\
& \dot{Y}_{f} \sin \varphi+\dot{X}_{f} \cos \varphi-b \dot{\varphi}=r \dot{\theta}_{l}
\end{aligned}
$$


The compact form of the nonholonomic constraints can be written as

$$
\mathbf{A}(\mathbf{q}) \dot{\mathbf{q}}=\mathbf{0}
$$

and

$$
\mathbf{A}=\left[\begin{array}{cccccccccc}
-\sin \varphi & \cos \varphi & -d & 0 & 0 & 0 & 0 & 0 & \ldots & 0 \\
-\cos \varphi & -\sin \varphi & -b & r & 0 & 0 & 0 & 0 & \ldots & 0 \\
-\cos \varphi & -\sin \varphi & b & 0 & r & 0 & 0 & 0 & \ldots & 0
\end{array}\right]
$$

where $r$ is the radius of the driving wheel, $b$ is the distance of two wheels and $d$ is the distance between the front and rear wheels.

By defining the matrix $\mathbf{B}(\mathbf{q})$, which is the null space of the matrix $\mathbf{A}(\mathbf{q})$, the Lagrange multipliers can be eliminated

$$
\mathbf{A}(\mathbf{q}) \mathbf{B}(\mathbf{q})=0
$$

One choice of $\mathbf{B}(\mathbf{q})$ is as follows

$$
\mathbf{B}=\left[\begin{array}{ccccccc}
(r / 2 b)(b \cos \varphi-d \sin \varphi) & (r / 2 b)(b \cos \varphi+d \sin \varphi) & 0 & 0 & 0 & \ldots & 0 \\
(r / 2 b)(b \sin \varphi+d \cos \varphi) & (r / 2 b)(b \sin \varphi-d \cos \varphi) n & 0 & 0 & 0 & \ldots & 0 \\
(r / 2 b) & -(r / 2 b) & 0 & 0 & 0 & \ldots & 0 \\
1 & 0 & 0 & 0 & 0 & \ldots & 0 \\
0 & 1 & 0 & 0 & 0 & \ldots & 0 \\
0 & 0 & 1 & 0 & 0 & \ldots & 0 \\
0 & 0 & 0 & 1 & 0 & \ldots & 0 \\
0 & 0 & 0 & 0 & 1 & \ldots & 0 \\
\vdots & \vdots & \vdots & \vdots & \vdots & \vdots & 0 \\
0 & 0 & 0 & 0 & 0 & 0 & 1
\end{array}\right]
$$

as well as $\dot{\mathbf{q}}$ can be expressed as follows

$$
\dot{\mathbf{q}}=\mathbf{B}(\mathbf{q}) \mathbf{v}
$$

where

$$
\mathbf{v}=\left[\dot{\theta}_{r}, \dot{\theta}_{l}, \dot{\theta}_{1}, \dot{\theta}_{2}, \dot{q}_{f 1}, \ldots, \dot{q}_{f n}\right]^{\mathrm{T}}
$$

By differentiating equation (2.12)

$$
\ddot{\mathbf{q}}=\mathbf{B}(\mathbf{q}) \dot{\mathbf{v}}+\dot{\mathbf{B}}(\mathbf{q}) \mathbf{v}
$$

after performing some algebraic manipulations, the dynamic equation of the mobile flexible manipulator is

$$
\mathbf{B}^{\mathrm{T}}(\mathbf{q}) \mathbf{M}[\mathbf{B}(\mathbf{q}) \dot{\mathbf{v}}+\dot{\mathbf{B}}(\mathbf{q}) \mathbf{v}]+\mathbf{B}^{\mathrm{T}}(\mathbf{q})(\mathbf{C}+\mathbf{G})=\mathbf{B}^{\mathrm{T}}(\mathbf{q}) \mathbf{F}
$$

Finally, the dynamic equations in the state space are as follow

$$
\dot{\mathbf{x}}=\left[\begin{array}{c}
\mathbf{B} \mathbf{v} \\
\left(\mathbf{B}^{\mathrm{T}} \mathbf{M B}\right)^{-1}\left(-\mathbf{B}^{\mathrm{T}} \mathbf{M} \dot{\mathbf{B}} \mathbf{v}-\mathbf{B}^{\mathrm{T}} \mathbf{C}\right)
\end{array}\right]+\left[\begin{array}{c}
\mathbf{0} \\
\left(\mathbf{B}^{\mathrm{T}} \mathbf{M B}\right)^{-1}
\end{array}\right] \mathbf{F}
$$

By using these equations, the optimal trajectory planning problem can be formulated. 


\section{Optimization strategy}

Path planning in the case of a mobile flexible manipulator is complex due to flexibility of the manipulator arm. In this Section, an indirect solution to the optimal control problem is applied for the off-line global trajectory planning of the mobile flexible manipulator. The purpose of the optimal control problem is to determine the control $u(t)$ that minimizes the performance index $J(u)$. In this investigation, the specific objective functional $J$ is to obtain the optimal paths with minimum effort and vibration. The general expression which minimizes the cost functional means that (Wang et al., 2001)

$$
\min J=\int_{t_{0}}^{t_{f}} L(\mathbf{X}(t), \mathbf{U}(t), t) d t=\frac{1}{2}\left\|\mathbf{X}_{1}\right\|_{\mathbf{W}_{P}}^{2}+\frac{1}{2}\left\|\mathbf{X}_{2}\right\|_{\mathbf{W}_{V}}^{2}+\frac{1}{2}\|\mathbf{U}\|_{R}^{2}
$$

Here, the integrand $L(\cdot)$ is a smooth differentiable function in the arguments, $\mathbf{X}(t)$ and $\mathbf{U}(t)$ denote the state space form of the generalized coordinate and the joint torque, respectively. $\|\mathbf{X}\|_{\mathbf{K}}^{2}=\mathbf{X}^{\mathrm{T}} \mathbf{K X}$ is the generalized squared norm, $\mathbf{W}_{P}, \mathbf{W}_{V}$ are symmetric, positive semi-definite $(k \times k)$ weighting matrix, and $\mathbf{R}$ is the symmetric, positive definite $(k \times k)$ matrix. The designer can decide on the relative importance among the angular position, angular velocity, vibration amplitude and control effort by the numerical choice of penalty matrices $\mathbf{W}_{P}, \mathbf{W}_{V}$ and $\mathbf{R}$. In order to minimize the objective function subjected to the nonlinear dynamic equations, the well-known Pontryagin minimum principle is used. By introducing the cost vector $\psi$, the Hamiltonian function of the system can be defined as

$$
H(\mathbf{X}, \mathbf{U}, \boldsymbol{\psi}, t)=L(\mathbf{X}, \mathbf{U})+\boldsymbol{\psi}^{\mathrm{T}} \dot{\mathbf{X}}
$$

The PMP then implies that the necessary condition for a local minimum is that $H$ be minimized with respect to $u(t)$ at all times. If it is assumed that the set of admissible inputs is bounded $U_{i}^{-} \leqslant u_{i}^{*} \leqslant U_{i}^{+}$, this condition is equivalent to

$$
\dot{\mathbf{X}}=\frac{\partial H}{\partial \boldsymbol{\psi}} \quad \dot{\boldsymbol{\psi}}=\frac{-\partial H}{\partial \mathbf{X}} \quad \mathbf{0}=\frac{\partial H}{\partial \mathbf{U}}
$$

The considered boundary conditions are

$$
\mathbf{X}\left(t_{i}\right)=\mathbf{X}_{i} \quad \mathbf{X}\left(t_{f}\right)=\mathbf{X}_{f}
$$

where $\mathbf{X}\left(t_{i}\right)$ and $\mathbf{X}\left(t_{f}\right)$ represent positions and velocities of the links at the beginning and at the end of the maneuver. The optimal trajectory is then obtained by solving the $2 n$ differential equations

$$
\begin{aligned}
& \dot{x}^{*}(t)=\frac{\partial H}{\partial p}\left(x^{*}(t), u^{*}(t), p^{*}(t), t\right) \\
& \dot{p}^{*}(t)=-\frac{\partial H}{\partial x}\left(x^{*}(t), u^{*}(t), p^{*}(t), t\right) \\
& H\left(x^{*}(t), u^{*}(t), p^{*}(t), t\right) \leqslant H\left(x^{*}(t), u(t), p^{*}(t), t\right)
\end{aligned}
$$

The control values are limited with the upper and lower bounds. One of the most commonly used motors for actuating the joints of small and medium size mobile robots are permanent magnet DC motors. Typical speed-torque characteristics of DC motors in which the relationship between speed and torque is linear are defined as below (Wang et al., 2001)

$$
\mathbf{U}_{\text {allow }}^{(+)}=\mathbf{K}_{1}-\mathbf{K}_{2} \dot{\mathbf{q}} \quad \mathbf{U}_{\text {allow }}^{(-)}=-\mathbf{K}_{1}-\mathbf{K}_{2} \dot{\mathbf{q}}
$$


where

$$
\mathbf{K}_{1}=\left[\begin{array}{llll}
\tau_{s 1} & \tau_{s 2} & \ldots & \tau_{s m}
\end{array}\right]^{\mathrm{T}} \quad \mathbf{K}_{2}=\operatorname{diag}\left[\begin{array}{llll}
\frac{\tau_{s 1}}{\omega_{1}} & \frac{\tau_{s 2}}{\omega_{2}} & \ldots & \frac{\tau_{s m}}{\omega_{m}}
\end{array}\right]^{\mathrm{T}}
$$

the stall torque (torque generated by the motor when fully "ON" but unable to move) and no-load speed (output speed of the motor when running without load) are denoted by $\tau_{s}$ and $\omega_{m}$, respectively.

\subsection{Maximum payload algorithm}

The set of dynamic equations, the governing optimal control problem and the boundary conditions lead to the standard form of a two-point boundary value problem (TPBVP). The collocation method is one of the basic ways of solving TPBVP. The method iterates on the initial values of the co-state until the final boundary conditions are satisfied by the following desired accuracy

$$
h\left(\mathbf{X}\left(t_{f}\right), t_{f}\right)=\frac{1}{2}\left\|\mathbf{X}_{1}\left(t_{f}\right)-\mathbf{X}_{1 f}\right\|_{\mathbf{W}_{p}}^{2}+\frac{1}{2}\left\|\mathbf{X}_{2}\left(t_{f}\right)-\mathbf{X}_{2 f}\right\|_{\mathbf{W}_{V}}^{2} \leqslant \varepsilon
$$

In this Section, an algorithm is proposed to find the maximum payload shown in Fig. 3. The proposed method considers torque bounds and is based on increasing the payload until one point

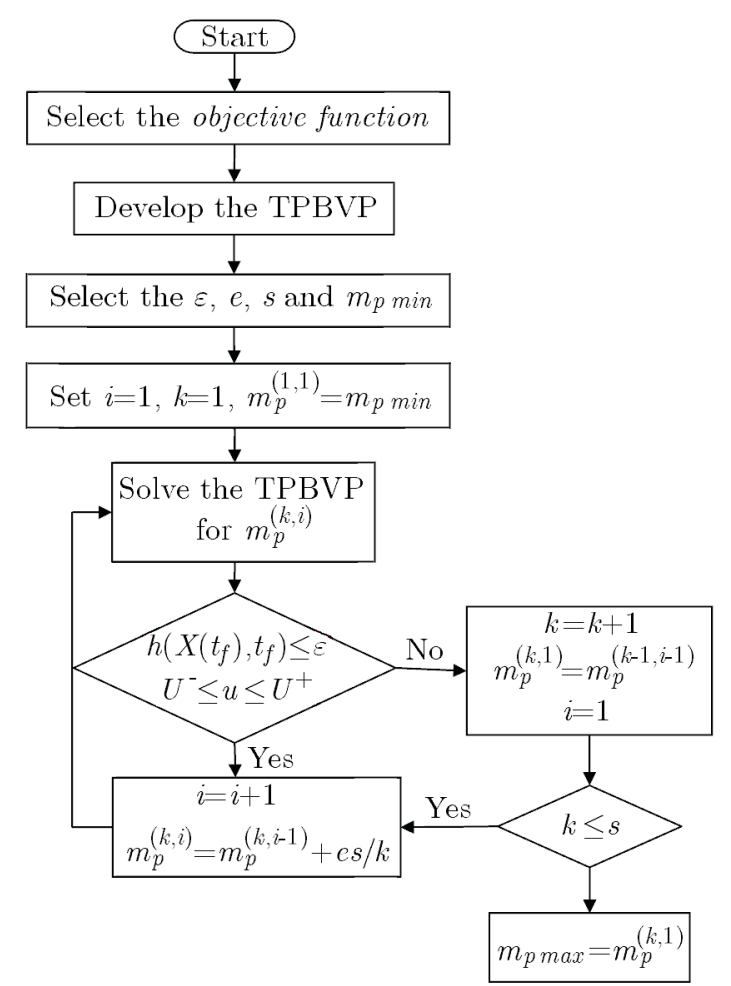

Fig. 3. The algorithm for calculation of the maximum payload

of one of the actuators torque reach the upper or lower torque bounds. As shown in Fig. 3, the proposed algorithm includes two stages; the first stage index $i$ increases the tip mass $m p$ until the actuators torque reach the upper or lower torque constraints. The desired accuracy $\varepsilon$ in TPBVP solution must be satisfied for the payload in each step. A further increase in the payload exceeds the torque limits. Consequently, the desired accuracy $\varepsilon$ in TPBVP solution could not be satisfied and the boundary conditions at yhe final time may be obtained incorrectly. At this status, while one point of one of the actuators torque reach the upper or lower torque bounds; the second stage 
index $k$ decreases the payload until the maximum payload for the supposed penalty matrices is obtained with the accuracy $\varepsilon$. The accuracy of the maximum payload $m p_{\max }$ calculation depends on the value $e$. The iteration number is denoted by $s$ symbol in this algorithm.

\subsection{Path planning in the presence of an obstacle}

In order for mobile flexible manipulators to successfully carry out tasks, especially in carrying heavy loads on different trajectories, the Artificial Potential Fields (APFs) is used throughout the robot workspace with each point in the workspace having an associated potential. The idea used in APF-based obstacle avoidance is to position a mobile manipulator in the workspace such that the overall potential encountered by the mobile manipulator is minimized while still accomplishing the desired task. A repulsive potential formulation based on the distance between parts of the WMM and obstacles is used in the cost function for obstacle avoidance. The most commonly used repulsive potential takes the form (Khatib, 1986)

$$
\mathbf{U}_{r e p}=\left\{\begin{array}{lll}
\frac{1}{2} \eta\left(\frac{1}{\rho\left(q, q_{o b s}\right)}-\frac{1}{\rho_{0}}\right) & \text { if } & \rho\left(q, q_{o b s}\right) \leqslant \rho_{0} \\
0 & \text { if } & \rho\left(q, q_{o b s}\right)>\rho_{0}
\end{array}\right.
$$

where $\eta$ is a positive weighting matrix, $\rho\left(q, q_{o b s}\right)$ denotes the minimal distance from the robot $q$ to the obstacle, $q_{\text {obs }}$ denotes the point on the obstacle such that the distance between this point and the robot is minimal between the obstacle and the robot, and $\rho_{0}$ is a positive constant denoting the distance of influence of the obstacle.

The obstacle avoidance problem is formulated in terms of collision avoidance of the base, links and joints with the obstacles. In order to add the penalty function to the performance index in order to guarantee free-collision motion of the mobile body, the distance between the center of the mobile base and the center of the obstacle will be

$$
\rho_{b}=\left\|\mathbf{P}_{o b s}-\mathbf{P}_{b}\right\|=\sqrt{\left(\mathbf{X}_{o b s}-\mathbf{X}_{b}\right)^{2}+\left(\mathbf{Y}_{o b s}-\mathbf{Y}_{b}\right)^{2}}
$$

By assuming the links as lines, the minimal distance between $i j$ link and the center of the obstacle can be calculated as

$$
\rho_{i j}=\frac{1}{\sqrt{\left(\mathbf{X}_{j}-\mathbf{X}_{i}\right)^{2}+\left(\mathbf{Y}_{j}-\mathbf{Y}_{i}\right)^{2}}}\left|\operatorname{det}\left[\begin{array}{cc}
\mathbf{X}_{j}-\mathbf{X}_{i} & \mathbf{X}_{o b s}-\mathbf{X}_{i} \\
\mathbf{X}_{j}-\mathbf{X}_{i} & \mathbf{Y}_{o b s}-\mathbf{Y}_{i}
\end{array}\right]\right|
$$

The position of parts in the workspace is denoted by $\mathbf{X}_{i}$ and $\mathbf{Y}_{i}$. Therefore, the objective function to guarantee the free-collision motion can be defined as

$$
J(u)=\int_{t_{0}}^{t_{f}} L(\mathbf{X}, \mathbf{U}, t) d t=\frac{1}{2}\left\|\mathbf{X}_{1}\right\|_{\mathbf{W}_{1}}^{2}+\frac{1}{2}\left\|\mathbf{X}_{2}\right\|_{\mathbf{W}_{2}}^{2}+\frac{1}{2}\|\mathbf{U}\|_{\mathbf{R}}^{2}+\frac{1}{2}\left\|\frac{1}{\boldsymbol{\rho}}-\frac{1}{\boldsymbol{\rho}_{0}}\right\|_{\eta}^{2}
$$

The new objective function is used to obtain the trajectory optimization problem to avoid collision of the WMM parts with the obstacles. Figure 4 shows the wheeled mobile robot in the presence of an obstacle.

\section{Simulation results}

A simulation study has been carried out to investigate further the validity and effectiveness of the mobile flexible manipulators in finding the optimal path between two points with different objective functions. A two-link planar manipulator is considered. It is mounted on a differentially driven mobile base at point $F$ on the main axis of the base (Fig. 4). The parameters of the mobile flexible manipulator are given in Table 1. 


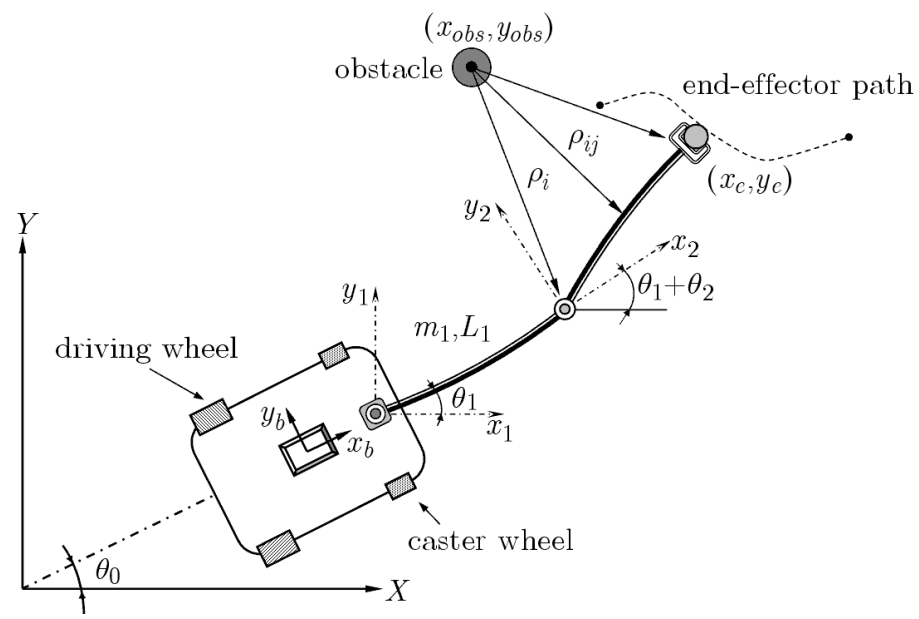

Fig. 4. WMM in the presence of an obstacle

Table 1. Simulation of parameters

\begin{tabular}{|l|c|c|}
\hline \multicolumn{1}{|c|}{ Parameter } & Value (base) & Value (manipulator) \\
\hline \hline Length $[\mathrm{m}]$ & $L_{0}=0.4$ & $L_{1}=L_{2}=1$ \\
\hline Mass $[\mathrm{kg}]$ & $m_{b}=94$ & $m_{1}=m_{2}=3, m p=0.5$ \\
\hline Cross section area $\left[\mathrm{m}^{2}\right]$ & - & $A_{1}=A_{2}=4 \cdot 10^{-8}$ \\
\hline Moment of inertia $\left[\mathrm{m}^{4}\right]$ & $I_{b}=6.609$ & $I_{1}=0.416, I_{2}=0.0625$ \\
\hline Young's modulus of the material $\left[\mathrm{N} / \mathrm{m}^{2}\right]$ & - & $E_{1}=E_{2}=2 \cdot 10^{10}$ \\
\hline
\end{tabular}

\subsection{First case study: optimal path for minimum effort}

The motion planning problem is to find the optimal trajectory with minimum effort. The main motivation behind the minimum effort is to find a path to reduce the amount of torques and hence to lower energy consumption. According to the algorithm presented in Fig. 3, the general solution method is based on increasing the payload from its minimum value $m p_{\min }$ up to the maximum payload can be found. Therefore, in this case, the initial payload, initial values of the co-state vector, accuracy values, and penalty matrices are considered as follows

$$
\begin{array}{llll}
m p_{\text {min }}=0.5 \mathrm{~kg} & \psi(0)=0 & e=0.1 & \varepsilon=0.0001 \\
\mathbf{W}_{p}=\mathbf{W}_{V}=\mathbf{0} & \mathbf{R}=\operatorname{diag}(1) & &
\end{array}
$$

This cost function is typical for systems that need to conserve energy during a particular operation. The actuator constants are given as follows

$$
\begin{aligned}
& \mathbf{K}_{1}=\left[\begin{array}{llll}
20 & 20 & 50 & 50
\end{array}\right]^{\mathrm{T}} \mathrm{N} \cdot \mathrm{m} \\
& \mathbf{K}_{2}=\operatorname{diag}\left[\begin{array}{llll}
1.5 & 1.5 & 2.5 & 2.5
\end{array}\right] \frac{\mathrm{N} \cdot \mathrm{m} \cdot \mathrm{s}}{\mathrm{rad}}
\end{aligned}
$$

The system is initially at rest, thus the mobile base is initially at the point $\left(x_{F}=0.75 \mathrm{~m}\right.$, $\left.y_{F}=-0.5 \mathrm{~m}, \varphi=0^{\circ}\right)$ and moves to its final position $\left(x_{F}=1.6 \mathrm{~m}, y_{F}=-0.2 \mathrm{~m}, \varphi=15^{\circ}\right)$. The initial conditions of the manipulator are $\theta_{1}(0)=1.5 \mathrm{rad}, \theta_{2}(0)=2 \mathrm{rad}, \dot{\theta}_{1}(0)=0, \dot{\theta}_{2}(0)=0$ (point $A$ in Fig. 5a) and the final conditions are $\theta_{1}\left(t_{f}\right)=-0.86 \mathrm{rad}, \theta_{2}\left(t_{f}\right)=1.09 \mathrm{rad}, \dot{\theta}_{1}\left(t_{f}\right)=0$, $\dot{\theta}_{2}\left(t_{f}\right)=0$ (point $B$ in Fig. 5a) during the overall time $t_{f}=1.9 \mathrm{~s}$ (see Fig. 5a) and also the remaining boundary conditions are equal to zero.

A comparative study is carried out between the rigid model and flexible models (linear and nonlinear models) as shown in Fig. 5b. The optimal angular positions of the link and wheels, corresponding to the minimum effort are shown in Figs. 6a and 6b. 

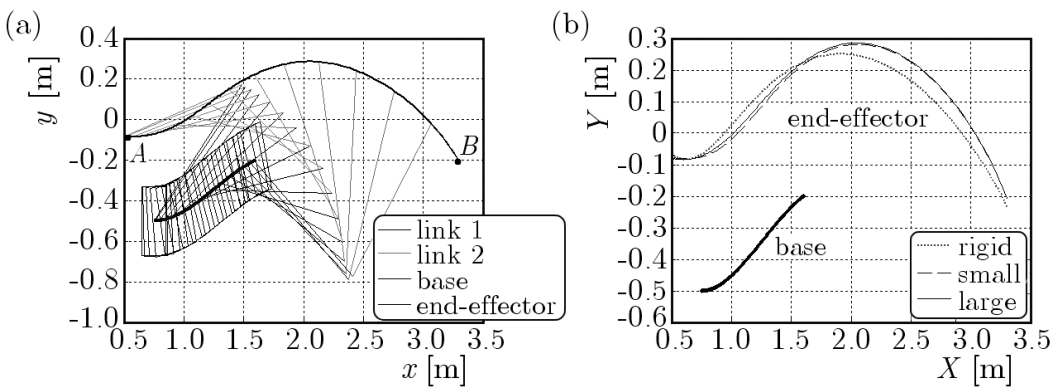

Fig. 5. The optimal paths between point $A$ and $B$ via minimum effort (a) and for different models (b)
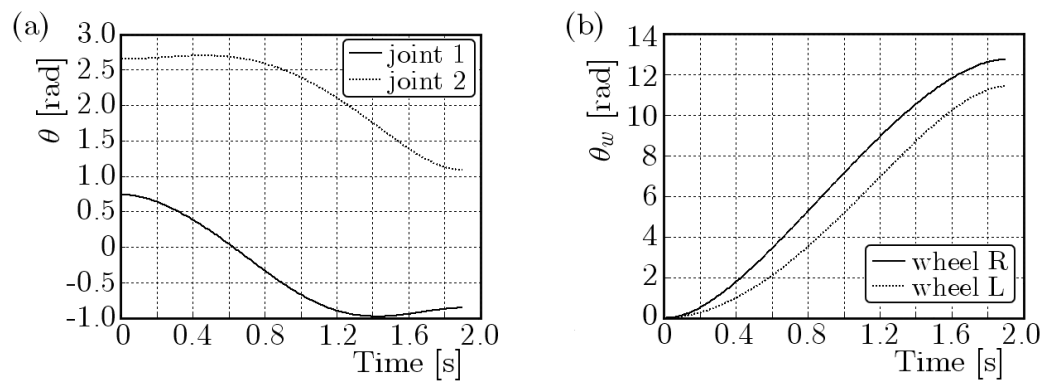

Fig. 6. The optimal angular positions of the first and second joints (a) and the right and left wheels (b)

The simulation results presented in Fig. 7 illustrate the optimal controls to carry the maximum payload, which also show the upper and lower bounds of the actuator torque capacity. By increasing the payload from $m p_{\min }$ to $m p_{\max }$, the required torque grows until one point of one of the actuators torque reach the upper or lower torque bounds. It can be seen that, the second motor reaches its maximum capacity. In this case study, the maximum payload is obtained to be $m p_{\max }=2.45 \mathrm{~kg}$, while by considering the rigid link and Nikoobin's method (Korayem et al., 2012 ), the maximum payload is found to be $m p_{\max }=8.25 \mathrm{~kg}$. This difference is due to flexibility of the link which increases the oscillation of torque curves.
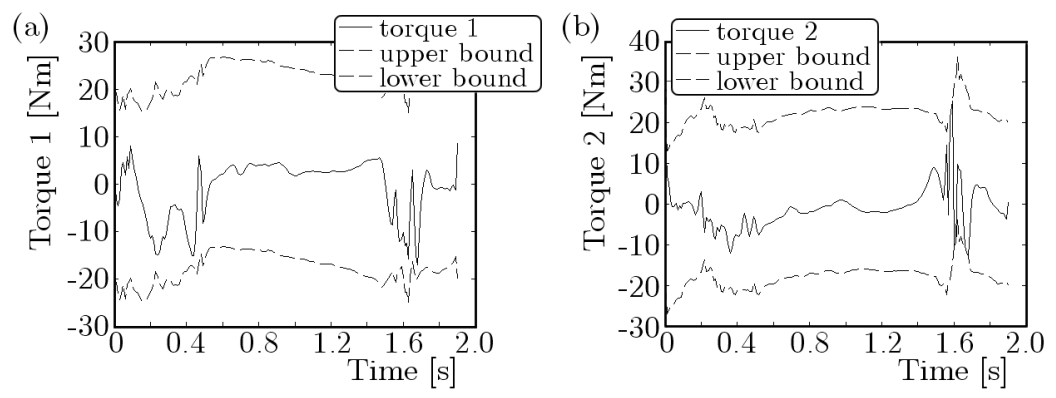

Fig. 7. Minimum effort of the first and second motors within upper and lower acceptable boundaries

\subsection{Second case study: minimum vibration trajectory}

In the motion planning of flexible robots, obtaining the minimum vibration trajectory is one of the most frequently encountered problems. The optimization objective is to minimize the vibration excitation during motion. By increasing the weighting factors corresponding to the derivative of flexural displacements $\left(\dot{q}_{f 1}, \ldots, \dot{q}_{f n}\right)$, the vibrational motions will be suppressed. The bounds of the motor capacity are not considered. Hence, the proper penalty matrices are selected to be $\mathbf{R}=\operatorname{diag}(0.01)$ and $\mathbf{W}_{p}=\mathbf{0}, \mathbf{W}_{V}=\operatorname{diag}(0,0,1, \ldots, 1)$. The load must be carried from the initial point with coordinate $\left(x_{e}=0.5 \mathrm{~m}, y_{e}=-0.08 \mathrm{~m}\right)$ to the final point with 
coordinate $\left(x_{e}=3.31 \mathrm{~m}, y_{e}=-0.25 \mathrm{~m}\right)$. The optimal trajectory between these two points during the overall time $t_{f}=1.9 \mathrm{~s}$ is desired for the rest-to-rest maneuver. The other conditions remain the same with the previous case study. The characteristics of the parts are shown in Table 1.

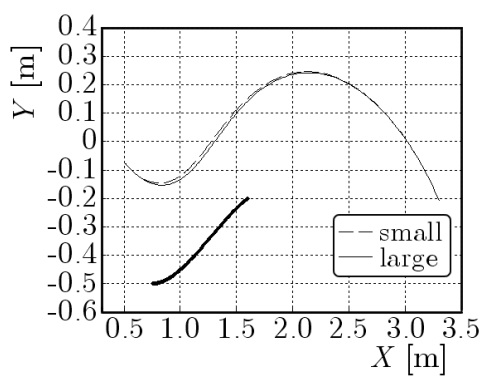

Fig. 8. The optimal paths via minimum vibration
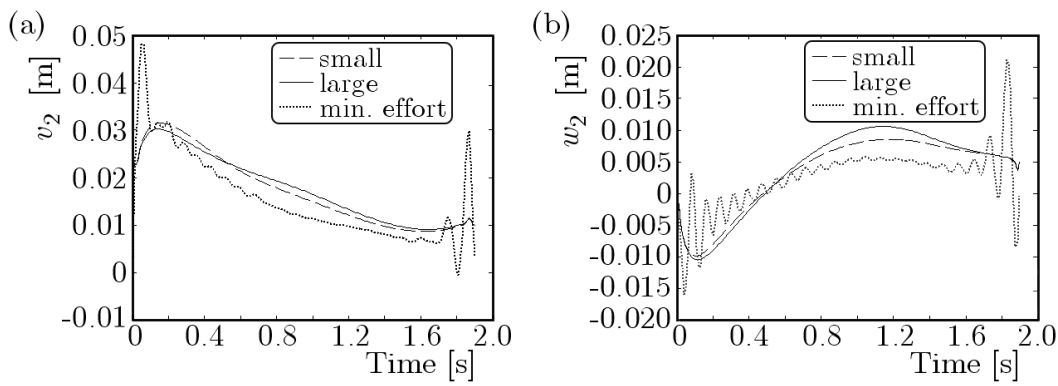

Fig. 9. The optimal flexural deflections of the first link (a) and the optimal flexural displacements of the second link (b)

The simulation results (small and large models) are illustrated in Fig. 8. The obtained optimal flexural deflections, corresponding to the minimum vibration are shown in Figs. 9a and 9b. The simulation results show that a significant reduction in manipulator vibration can be achieved by employing the proposed optimization procedure. As expected, it can be seen, by decreasing the amplitude vibration, that the linear and nonlinear models come closer together. Also, it is shown that application of the proper input torque may decrease the end effector vibration significantly.

\subsection{Third case study: trajectory optimization in the presence of an obstacle}

Recently, there has been a great deal of interest in path planning for autonomous mobile manipulators in the presence of an obstacle because of their ability to replace objects in a wide workspace. Hence, the problem is how to find a feasible trajectory for all components in order to carry the maximum payload in environment with an obstacle. The characteristics of the nonholonomic mobile manipulator, penalty matrices, accuracy value, and the actuator constants are the same as in the first case study. Moreover, the augmented functional obstacle avoidance is considered in the cost function. The task is considered to move the end effector from the initial point $p_{0}=(0.5,-0.08)$ to the final configuration $p_{f}=(3.31,-0.25)$ for the rest-to-rest maneuver during the overall time $t_{f}=1.9 \mathrm{~s}$. Also, there is an obstacle with $r_{\text {obs }}=0.05 \mathrm{~m}$ at a point with coordinates $p_{o b s}=\left(x_{o b s}=1.1 \mathrm{~m}, y_{o b s}=-0.55 \mathrm{~m}\right)$. The simulation parameters are shown in Table 1.

In this condition, the maximum payload is found to be $2.05 \mathrm{~kg}$. To avoid the obstacle, the manipulator moves far from it and causes a decrease in the allowable payload. The schematic of the obstacle and the obtained optimal paths of the end-effector and the mobile base are shown in Fig. 10a. A comparative study is performed between the rigid model and flexible models (linear and nonlinear models) as shown in Fig. 10b. 

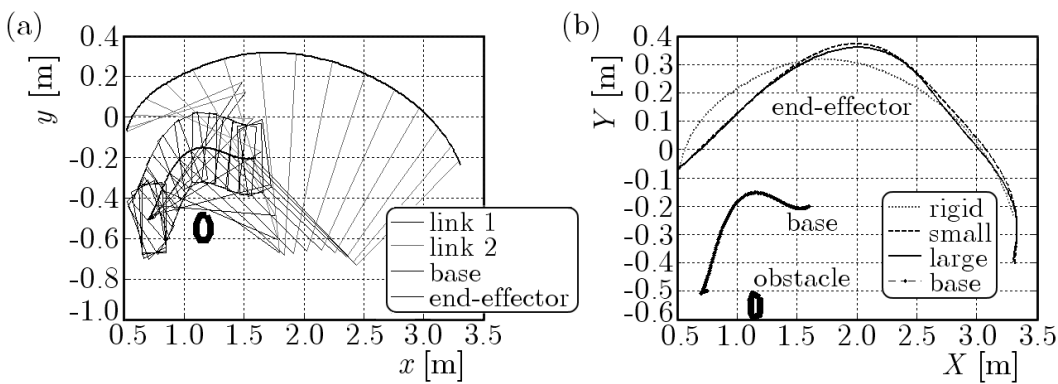

Fig. 10. The schematic view of obtained optimal paths in the presence of an obstacle (a) and the obtained optimal paths of the end-effector and mobile base for different models (b)

\section{Conclusions}

The main objective of this investigation is to determine the trajectory optimization for calculating the MADL of flexible-link mobile manipulators in the point-to-point maneuver in the presence of an obstacle, based on the indirect solution to the optimal control problem. The effect of dynamic interaction between the flexible manipulator and the mobile platform is considered to characterize the motion of a nonholonomic mobile manipulator with the compliant link capable of large deflection, in which both the geometric elastic nonlinearity and the foreshortening effects are considered. This model leads to a constant stiffness matrix and makes the formulation particularly efficient in computational terms and numerically more stable than alternative geometrically nonlinear formulations based on lower-order terms. Pontryagin's minimum principle is used to obtain the optimality conditions, which leads to a standard form of a two-point boundary value problem. An augmented objective function based on an artificial potential field is considered to avoid the obstacle during point-to-point maneuvers. Several simulation studies on a nonholonomic wheeled mobile manipulator are carried out for finding the MADL and optimal paths with different objective functions like minimum effort and minimum vibration. The numerical results indicate the effect of employing trajectory optimization in the performance improvement of the mobile flexible manipulator. It is shown that the presence of the obstacle causes the manipulator moves far from it; therefore, it reduces the maximum load-carrying capacity. Moreover, in this method, the designer can compromise between different objectives by considering proper penalty matrices, and may choose the proper trajectory between various paths. The obtained results illustrate the power and efficiency of the model in overcomming the highly nonlinear nature of the large deflection and optimization problem, which with other methods may be very difficult or impossible to achieve.

\section{References}

1. Agirrebeitia J., Aviles R., Ajuria G., 2005, A new APF strategy for path planning in environments with obstacles, Mechanism and Machine Theory, 40, 1, 645-658

2. Castro D., Nunes U., Ruano A., 2002, Obstacle avoidance in local navigation, Proceedings of the 10th Mediterranean Conference on Control and Automation, Lisbon, Portugal

3. Damaren C., Sharf L., 1995, Simulation of flexible-link manipulators with inertia and geometric nonlinearities, ASME Journal of Dynamic Systems, Measurement, and Control, 117, 1, 74-87

4. Кнатів O., 1986, Real-time obstacle avoidance for manipulators and mobile robots, The International Journal of Robotics Research, 5, 1, 90-98

5. Korayem M.H., Haghpanahi M., Heidari H.R., 2012, Analysis of flexible mobile manipulators undergoing large deformation with stability consideration, Latin American Applied Research, 42, 2 
6. PArk K.J., 2003, Path design of redundant flexible robot manipulators to reduce residual vibration in the presence of obstacles, Robotica, 21, 1, 335-340

7. Seraji H., 1998, A unified approach to motion control of mobile manipulators, The International Journal of Robotics Research, 17, 12, 107-118

8. Wang C.Y.E., Timoszyk W.K., Bobrow J.E., 2001, Payload maximization for open chained manipulator: finding weightlifting motions for a Puma 762 robot, IEEE Transactions on Robotics and Automation, 17, 1, 218-224

9. Xi F., Fenton R.G., 1991, A quasi-static motion planner for flexible manipulators using the algebra of rotations, IEEE International Conference on Robotics and Automation, 3, 1, 2363-2368

10. Yамамото Y., Yun X., 1994, Coordinating locomotion and manipulation of a mobile manipulator, IEEE Transactions on Robotics and Automation, 39, 6, 1326-1332

Manuscript received March 12, 2015; accepted for print May 8, 2015 